Research Article

\title{
Numerical Study of Disturbance Resistance of Oblique Detonation Waves
}

\author{
Yu Liu $(\mathbb{D}$, Baoguo Xiao $(\mathbb{D}$, Lan Wang, and Chao Wang \\ Science and Technology on Scramjet Laboratory, China Aerodynamics Research and Development Center (CARDC), \\ Sichuan Mianyang 621000, China \\ Correspondence should be addressed to Baoguo Xiao; xiaobaoguo@cardc.cn
}

Received 23 July 2020; Revised 31 October 2020; Accepted 17 November 2020; Published 2 December 2020

Academic Editor: Zhaoxin Ren

Copyright ( $2020 \mathrm{Yu}$ Liu et al. This is an open access article distributed under the Creative Commons Attribution License, which permits unrestricted use, distribution, and reproduction in any medium, provided the original work is properly cited.

\begin{abstract}
The stability of oblique detonation waves (ODWs) is a fundamental problem, and resistance of ODWs against disturbances is crucial for oblique detonation engines in high-speed propulsion. In this work, numerical studies on ODW stability in disturbed flows are conducted using the two-dimensional reactive Euler equations with a two-step induction-reaction kinetic model. Two kinds of flow disturbances are, respectively, introduced into the steady flow field to assess ODW stability, including upstream transient high-pressure disturbance (UTHD) and downstream jet flow disturbance (DJFD) with different durations. Generally, an ODW is susceptible to disturbances at larger wedge angles and stable at smaller wedge angles. In the unstable wedge angle range, different ODW structures and transition patterns are obtained after disturbances, including different locations of the primary triple points, different numbers of the steady triple points on the wave surface, and different transition patterns from the leading oblique shock wave to the ODW. It is found that the primary triple point tends to move upstream for the disturbances that can form a local strong detached bow shock wave near the wedge tip. In contrast, the wave surface and the transition pattern are susceptible to all of the disturbances introduced in this study. Despite the unstable responses of the ODWs to the disturbances, the ODWs can keep standing stability after disturbances, which is beneficial to the propulsion application of ODWs.
\end{abstract}

\section{Introduction}

Standing stability of ODWs is important for oblique detonation engines because it involves the realization of its advantages in hypersonic propulsion, including fast heat release and high thermodynamic cycle efficiency. A standing ODW should first satisfy the requirement of the wedge angle according to Pratt et al. [1]; that is, the wedge angle should lie between the minimum value called the ChapmanJouguet (CJ) wedge angle and the maximum value called the detachment wedge angle. However, according to Ghorbanian and Sterling [2], the maximum allowable wedge angle should be less than the detachment wedge angle if a secondary CJ ODW forms following the leading shock. If the ODW initiation condition is not considered, the minimum wedge angle is not actually limited by the CJ wedge angle, according to Ashford and Emanuel [3]. In this situation, the ODW angle will remain constant at the $\mathrm{CJ}$ wave angle as the wedge angle decreases below the CJ wedge angle, with the Taylor rarefaction waves following the ODW itself. A series of studies on ODW structures [4-16] demonstrate ODW characteristics.

The aforementioned studies are based on twodimensional flow fields. If a conical shock wave is considered, the flow field will differ from the wedge flow to some extent due to the curvature effect and the presence of the TaylorMaccoll flow $[9,17,18]$. In addition to the wedge flow and conical flow, the spherical flow is another major concern for ODW initiation and standing stability. For the spherical flow, experiments on hypervelocity projectiles were comprehensively conducted by some researchers [19-23]. They conducted a series of parameter studies and investigated the criticality of stabilized ODWs around a spherical projectile. All of these results provide important benefits for ODW applications in propulsion. 
TABLE 1: Numerical parameter setup.

\begin{tabular}{|c|c|c|c|c|}
\hline Inflow Mach number, $M_{\infty}$ & $\begin{array}{l}\text { Inflow static temperature, } T_{\infty} \\
(\mathrm{K})\end{array}$ & $\begin{array}{c}\text { Inflow static pressure, } P_{\infty} \\
(\mathrm{pa})\end{array}$ & Specific heat ratio, $\gamma$ & $\begin{array}{l}\text { Dimensionless } \\
\text { heat release, } \tilde{Q}\end{array}$ \\
\hline 4.0 & 650 & 20000 & 1.2 & 8 \\
\hline $\begin{array}{l}\text { Dimensionless activation } \\
\text { energy of the induction step, } \\
E_{\mathrm{I}} / R T_{S}\end{array}$ & $\begin{array}{c}\text { Dimensionless activation } \\
\text { energy of the heat release } \\
\text { step, } E_{\mathrm{R}} / R T_{S}\end{array}$ & $\begin{array}{c}\text { Induction length } \\
\text { corresponding to a CJ } \\
\text { detonation, } \Delta I(\mathrm{~mm})\end{array}$ & $\begin{array}{l}\text { Thermal pulse width } \\
\text { corresponding to a } \mathrm{CJ} \\
\text { detonation, } \Delta R(\mathrm{~mm})\end{array}$ & $\begin{array}{c}\text { Gas constant, } \\
R(\mathrm{~J} / \mathrm{kg} \cdot \mathrm{K})\end{array}$ \\
\hline 4.8 & 1.0 & 2.12 & 0.95 & 377.9 \\
\hline
\end{tabular}

One feature of a stabilized ODW is its stability or resistance to disturbances. If an ODW cannot maintain standing stabilization when experiencing additional disturbances, propulsion using ODW will be meaningless because, during a flight process, changes in the inflow conditions and flow disturbances are usually inevitable. The previous studies on the ODW disturbance resistance examined responses of ODWs to transient disturbances inside the flow field (see $\mathrm{Li}$ et al. [4], Fusina et al. [24], Teng et al. [25], Liu et al. [12], and Miao et al. [26]) or to nonuniform or nonconstant boundary conditions (see Fang et al. [27] and Yang et al. [28]). Recently, the influence of geometric disturbances on ODWs has also been investigated $[29,30]$. For the transient disturbances, all of the papers indicated that an ODW is resilient to disturbances and can recover to its original state. However, Liu et al. [31] demonstrated the hysteresis phenomenon of the ODW recently. That means an ODW structure is not totally independent of the initial condition. ODW stability to inflow disturbances obtained by earlier studies $[4,12$, 24-26] was probably due to the selected small wedge angles for which the ODWs are inherently stable or because of the transient feature of the disturbances giving insufficient time to trigger instability. Therefore, this study will introduce different kinds of flow disturbances at different wedge angles to investigate when and how an ODW becomes unstable to disturbances.

\section{Numerical Treatment}

The governing equations to be numerically solved are the two-dimensional unsteady reactive Euler equations given as follows.

The continuity equation is

$$
\frac{\partial \rho}{\partial t}+\frac{\partial(\rho u)}{\partial x}+\frac{\partial(\rho v)}{\partial y}=0
$$

where $\rho, u, v, t, x$, and $y$ are the density, the $x$-direction velocity, the $y$-direction velocity, the time, the $x$ coordinate, and the $y$ coordinate, respectively.

The conservation equation of $x$-direction momentum is

$$
\frac{\partial(\rho u)}{\partial t}+\frac{\partial\left(\rho u^{2}+p\right)}{\partial x}+\frac{\partial(\rho u v)}{\partial y}=0
$$

where $p$ is the static pressure.

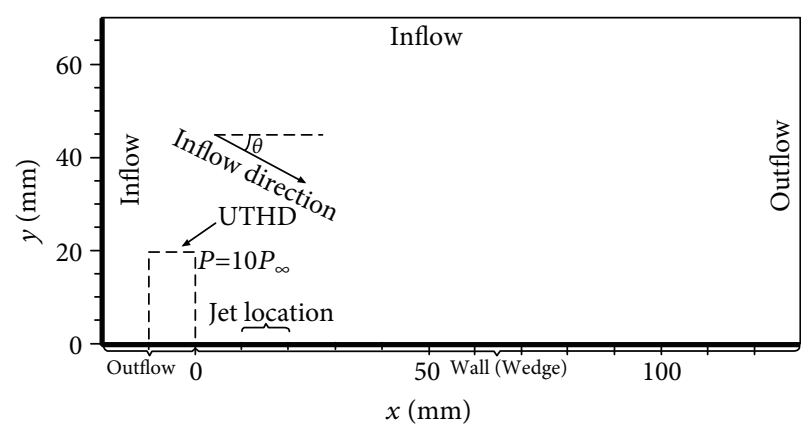

FIgURE 1: The computational domain.

The conservation equation of $y$-direction momentum is

$$
\frac{\partial(\rho v)}{\partial t}+\frac{\partial(\rho u v)}{\partial x}+\frac{\partial\left(\rho v^{2}+p\right)}{\partial y}=0 .
$$

The conservation equation of energy is

$$
\frac{\partial(\rho e)}{\partial t}+\frac{\partial[u(\rho e+p)]}{\partial x}+\frac{\partial[v(\rho e+p)]}{\partial y}=0
$$

where $e$ is the specific total energy and is described in Equation (6).

The equation of state for perfect gas is

$$
p=\rho R T
$$

where $R$ and $T$ are the gas constant and the static temperature, respectively. Thus, the specific total energy can be written as

$$
e=\frac{p}{\rho(\gamma-1)}+\frac{\left(u^{2}+v^{2}\right)}{2}-R T_{0} \tilde{q},
$$

where $\gamma, T_{0}$, and $\tilde{q}$ are the specific heat ratio, the static temperature of the oncoming flow, and the dimensionless local heat release (using $R T_{0}$ for normalization, i.e., $\tilde{q}=q / R T_{0}$, where $q$ is the dimensional local heat release), respectively.

A two-step induction-reaction kinetic model proposed by $\mathrm{Ng}$ et al. [32] and widely used in oblique detonation simulation $[14,33]$ consisting of a thermally neutral induction step and a heat release step is employed which allows us to easily change the induction length simply by modifying the 


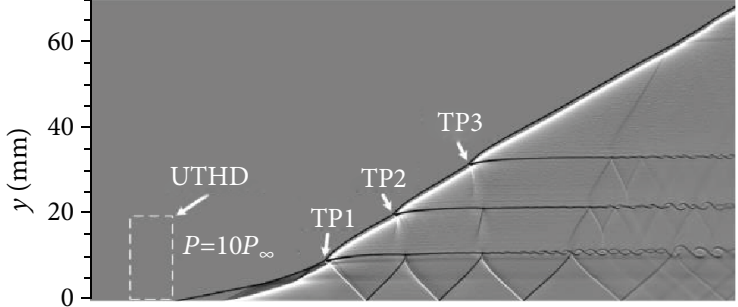

(a)

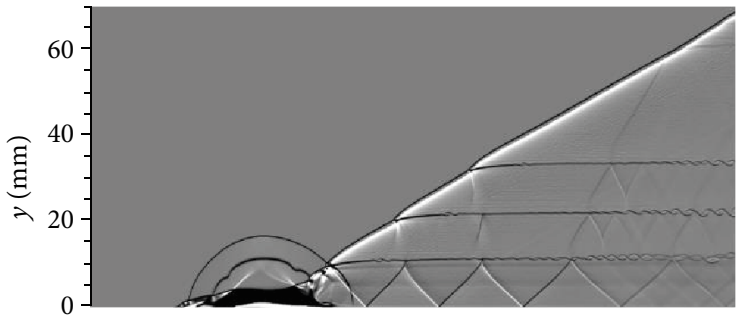

(b)

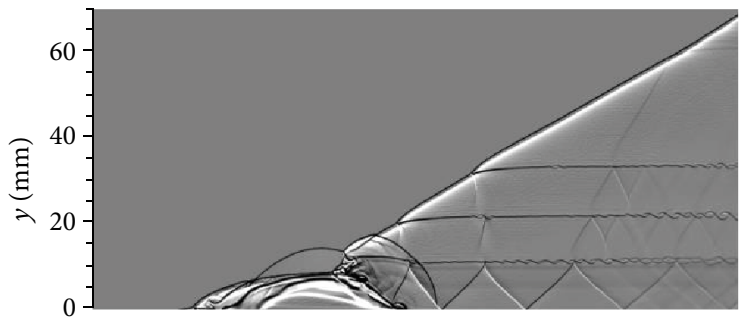

(c)

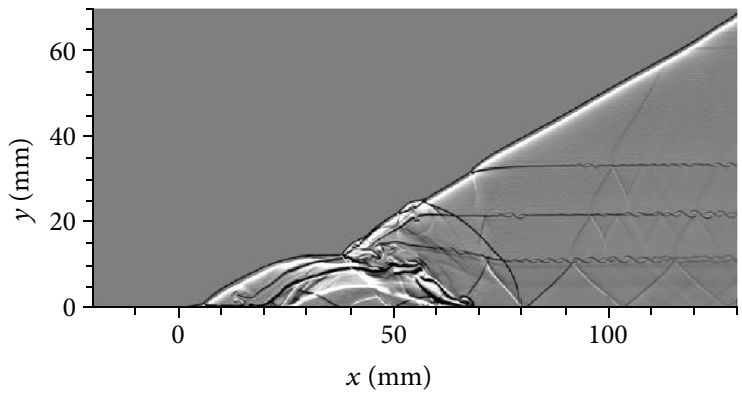

(d)

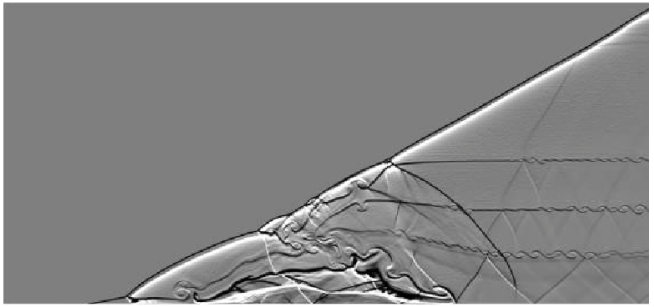

(e)

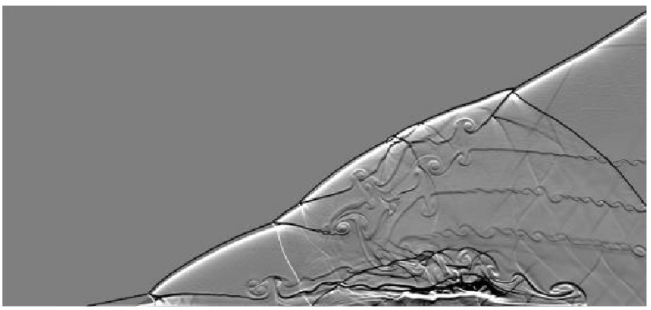

(f)

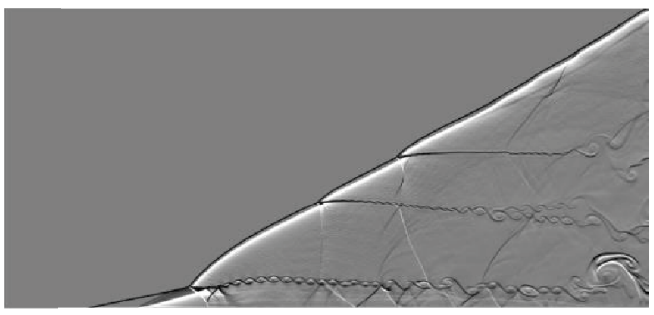

(g)

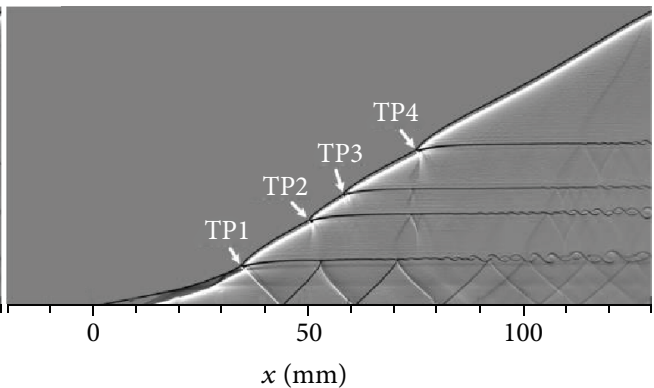

(h)

Figure 2: Evolution of the flow field after UTHD at $\theta=32^{\circ}$. (a) $t=0$ (the disturbance has just been introduced into the flow field), (b) $t=$ $200 \Delta t$, (c) $t=300 \Delta t$, (d) $t=400 \Delta t$, (e) $t=500 \Delta t$, (f) $t=700 \Delta t$, (g) $t=1500 \Delta t$, and (h) steady state after the disturbance.

reaction rate constant of the induction step. The reaction rate equations for both steps are given as

$\frac{\partial(\rho \xi)}{\partial t}+\frac{\partial(\rho u \xi)}{\partial x}+\frac{\partial(\rho v \xi)}{\partial y}=H(1-\xi) \rho k_{\mathrm{I}} \exp \left[E_{\mathrm{I}}\left(\frac{1}{R T_{S}}-\frac{1}{R T}\right)\right]$

$\frac{\partial(\rho \lambda)}{\partial t}+\frac{\partial(\rho u \lambda)}{\partial x}+\frac{\partial(\rho v \lambda)}{\partial y}=[1-H(1-\xi)] \rho k_{\mathrm{R}}(1-\lambda) \exp \left(\frac{-E_{\mathrm{R}}}{R T}\right)$

where $\xi, k_{\mathrm{I}}$, and $E_{\mathrm{I}}$ are the reaction progress variable (indicating the finishing degree of the current step), the reaction rate constant, and the activation energy of the induction step, respectively, and $\lambda, k_{\mathrm{R}}$, and $E_{\mathrm{R}}$ are that of the heat release step, respectively. Note that the reaction rate constants $k_{\mathrm{I}}$ and $k_{\mathrm{R}}$ control the induction length and the thermal pulse width, respectively. Usually, the induction length corresponding to a CJ detonation, $\Delta I$, and the thermal pulse width corresponding to a CJ detonation, $\Delta R$, are used to reflect the values of $k_{\mathrm{I}}$ and $k_{\mathrm{R}}$, respectively. In this study, the grid resolution satisfies that there are 32 grid points in $\Delta I$ (with the grid convergence test given in Section 3). $T_{S}$ is the temperature inside the induction region. $H(1-\xi)$ is a step function written as

$$
H(1-\xi)=\left\{\begin{array}{lll}
1 & \text { if } & \xi<1 \\
0 & \text { if } & \xi<1
\end{array}\right.
$$



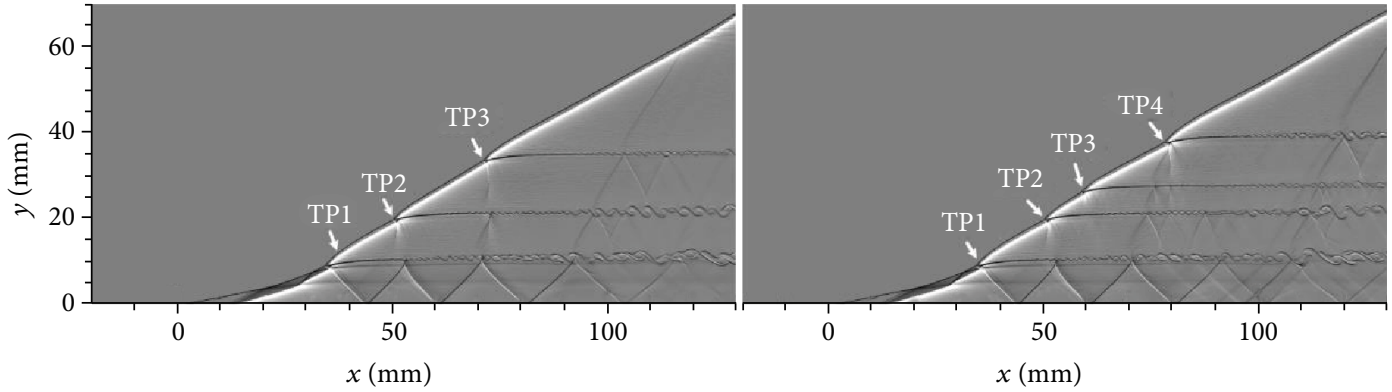

Figure 3: Grid convergence test at $\theta=32^{\circ}$. (a) Undisturbed steady flow field for $64 \mathrm{pts} / \Delta I$ and (b) steady flow field after UTHD for 64 pts/ $\Delta I$.

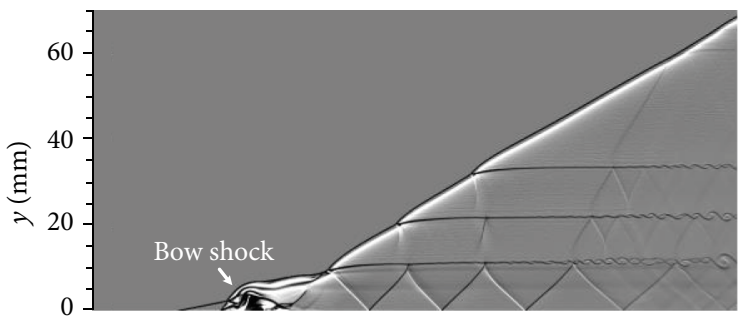

(a)

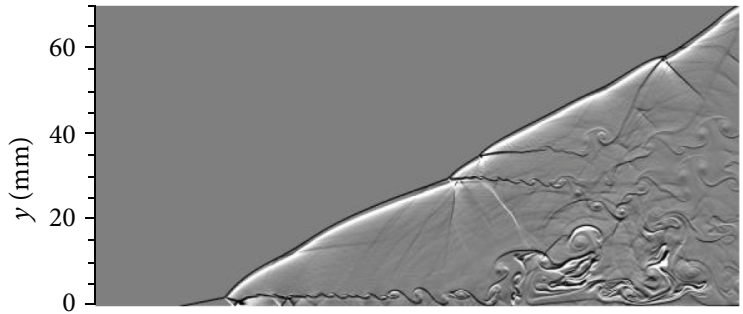

(b)

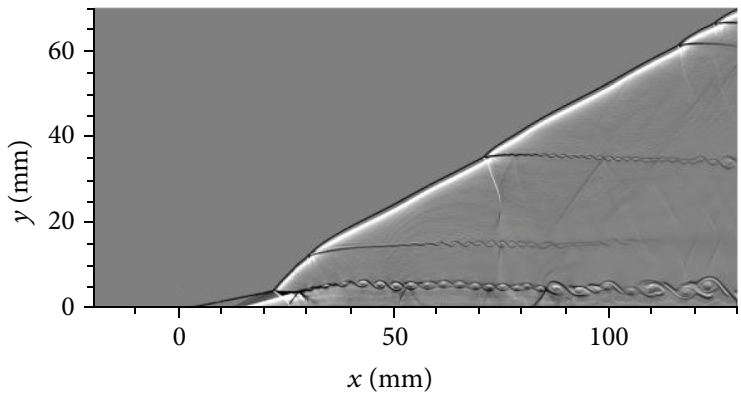

(c)

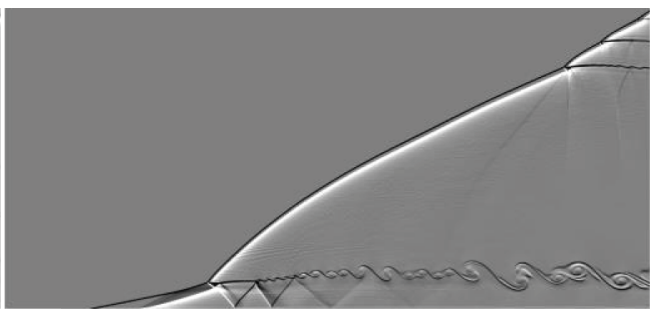

(d)

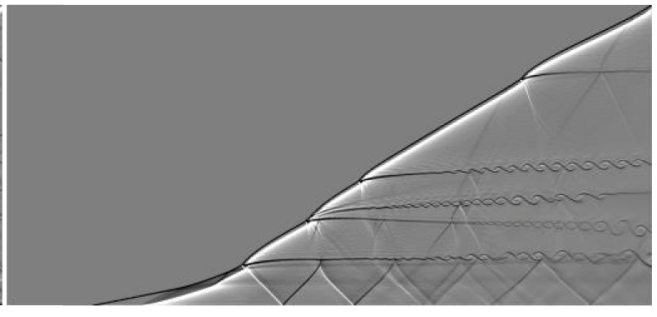

(e)

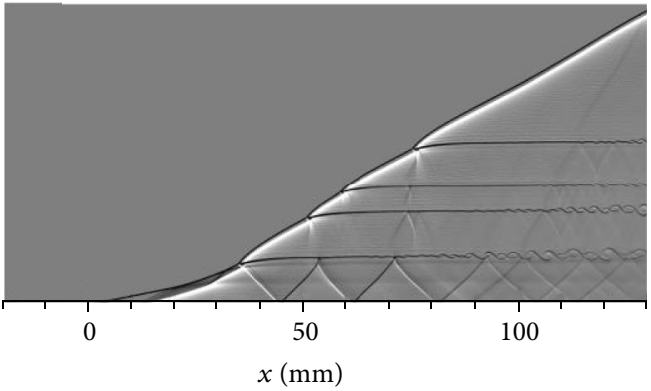

(f)

Figure 4: Evolution of the flow field after DJFD of $100 \Delta t$ at $\theta=32^{\circ}$. (a) $t=100 \Delta t$ (the jet flow has just been turned off), (b) $t=900 \Delta t$, (c) $t=2100 \Delta t$, (d) $t=5700 \Delta t$, (e) $t=16200 \Delta t$, and (f) steady state after the disturbance.

Clearly, at any instant, the local heat release $\tilde{q}$ or $q$ satisfies $\tilde{q}=\lambda \tilde{Q}$ or $q=\lambda Q$, where $\tilde{Q}$ and $Q$ are the dimensionless and dimensional total heat released by the reactant $\left(\tilde{Q}=Q / R T_{0}\right)$, respectively.

The governing equations above are numerically solved using an open-source adaptive mesh refinement computational fluid dynamic program called AMROC [34] which is based on the finite volume method. The block-structured adaptive mesh refinement technique of AMROC makes high-resolution capture of discontinuities in the flow field possible. Discretization is conducted using the MUSCL-
Hancock scheme which is second-order accurate, together with the hybrid Roe scheme as the Riemann solver. In order to ensure total variation diminishing (TVD) properties, a van Albada slope limiter is employed. The Godunov splitting method is used to treat the source term so as to overcome the problem of stiff chemistry. The parameter setup is listed in Table 1. The AMROC program with the two-step induction-reaction kinetic model has also been used by $\mathrm{Li}$ et al. $[35,36]$ to investigate detonation reflection. The computational domain is shown in Figure 1. The left and top boundaries are the inflow boundaries, and the bottom 


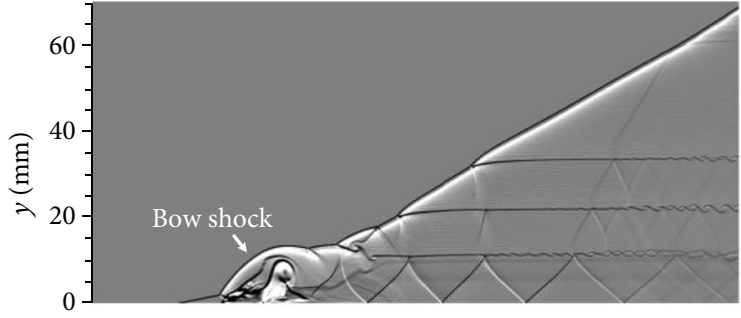

(a)

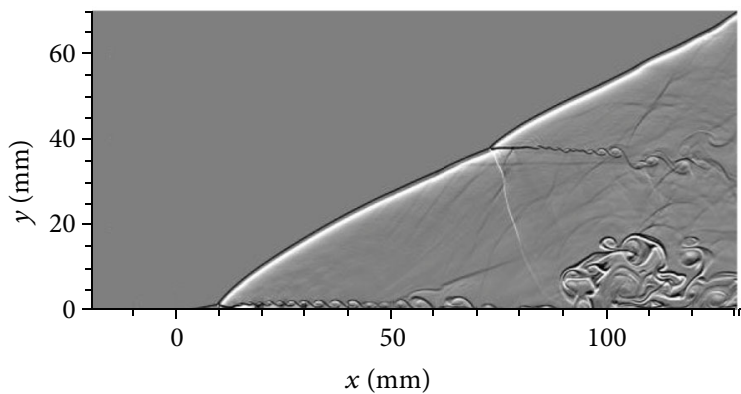

(b)

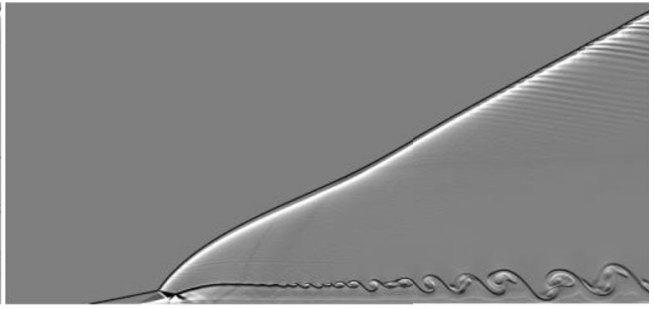

(c)

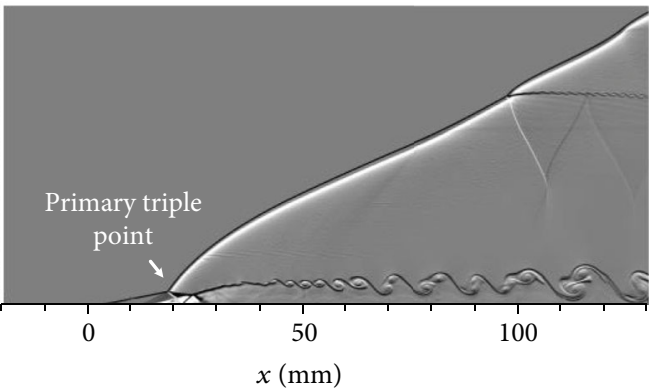

(d)

Figure 5: Evolution of the flow field after DJFD of $200 \Delta t$ at $\theta=32^{\circ}$. (a) $t=200 \Delta t$ (the jet flow has just been turned off), (b) $t=1200 \Delta t$, (c) $t=22800 \Delta t$, and (d) $t=36500 \Delta t$.

boundary in the range of $0 \mathrm{~mm} \leq x \leq 130 \mathrm{~mm}$ is a fixed wall. The angle between the inflow direction and the horizontal direction, $\theta$, represents the wedge angle. $\theta$ can vary so that different wedge angles can be tested. The right boundary and the bottom boundary in the range of $-20 \mathrm{~mm} \leq x<0$ $\mathrm{mm}$ are the outflow boundaries on which the flow field variants are extrapolated from the inside.

In this study, all the original steady flow fields are obtained through the following method: an inert shock wave is obtained by turning off the chemical reactions, and then an ODW is obtained by turning on the reactions in the cold flow. As introduced in Section 1, we will introduce different kinds of flow disturbances into the original steady flow fields. To be specific, the flow disturbances include an upstream transient high-pressure region (UTHD) and a downstream jet flow (DJFD). UTHD is within a rectangular zone of -10 $\mathrm{mm} \leq x<0 \mathrm{~mm}$ and $0 \mathrm{~mm} \leq y<20 \mathrm{~mm}$ where the local static pressure $P$ is 10 times the inflow static pressure $P_{\infty}$. DJFD is located in the range of $10 \mathrm{~mm} \leq x \leq 20 \mathrm{~mm}$ on the bottom boundary. The jet is a sonic flow normal to the bottom boundary, at a static pressure 10 times that of the inflow, and with other parameters the same as that of the inflow. In this study, the jet's lasting period is variable in different cases to check the effects, including 100 time steps, 200 time steps, and 500 time steps. The time step $\Delta t$ is controlled by the Courant number. In this study, the Courant number of 0.95 is used, which is corresponding to a time step of approximately $10^{-7}$ seconds. All of the results in this study are presented in the form of numerical schlieren, $\partial \rho / \partial y$.

\section{Results and Discussion}

Three different wedge angles including $\theta=32^{\circ}, \theta=31^{\circ}$, and $\theta=30^{\circ}$ are tested in this study. In the following, the results

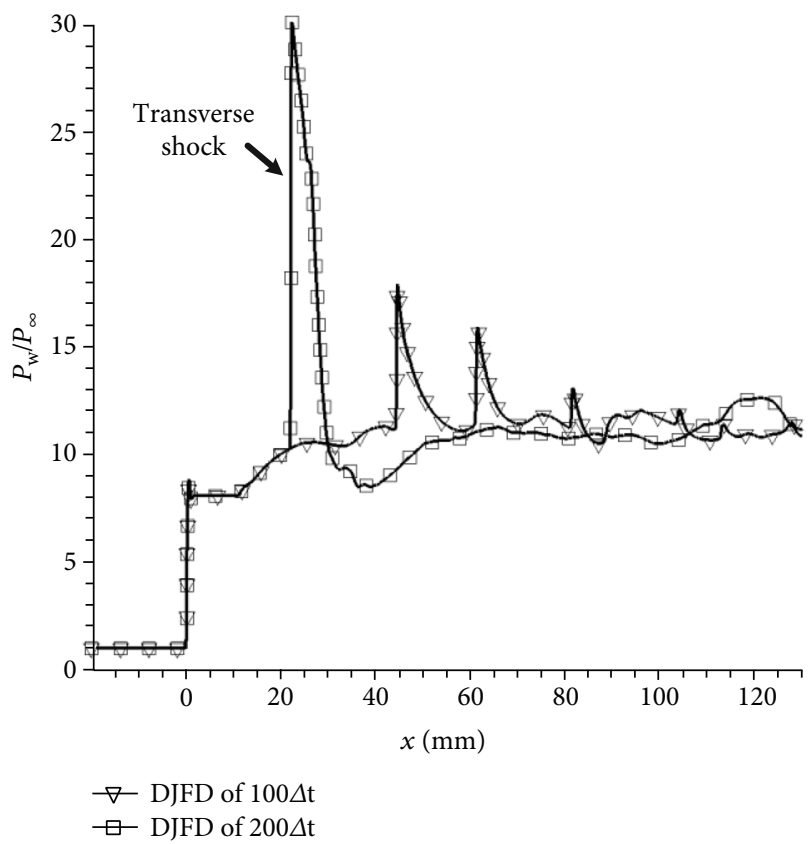

Figure 6: Pressure distributions on the bottom boundary of the steady flow fields after the DJFD of $100 \Delta t$ and $200 \Delta t$ at $\theta=32^{\circ}$.

of the $\theta=32^{\circ}$ case are given. Figure 2 shows the evolution of the flow field after UTHD. At $t=0$, a high-pressure disturbance region of $P=10 P_{\infty}$ is introduced in the upstream of the leading shock of the original steady ODW at $\theta=32^{\circ}$, as shown in Figure 2(a). In Figure 2(a), the wave surface of the ODW has three steady triple points, indicated by TP1, TP2, and TP3, respectively. TP1 is the primary triple point. Since TP1, TP2, and TP3 are all steady, they differ from the unstable triple points of cellular structures that are 


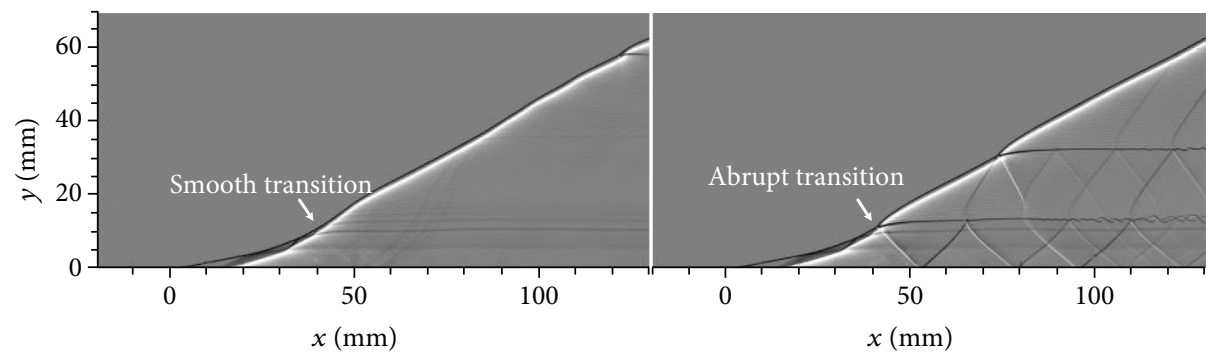

(a)

(b)

Figure 7: Steady flow fields before and after UTHD at $\theta=31^{\circ}$. (a) Before and (b) after.

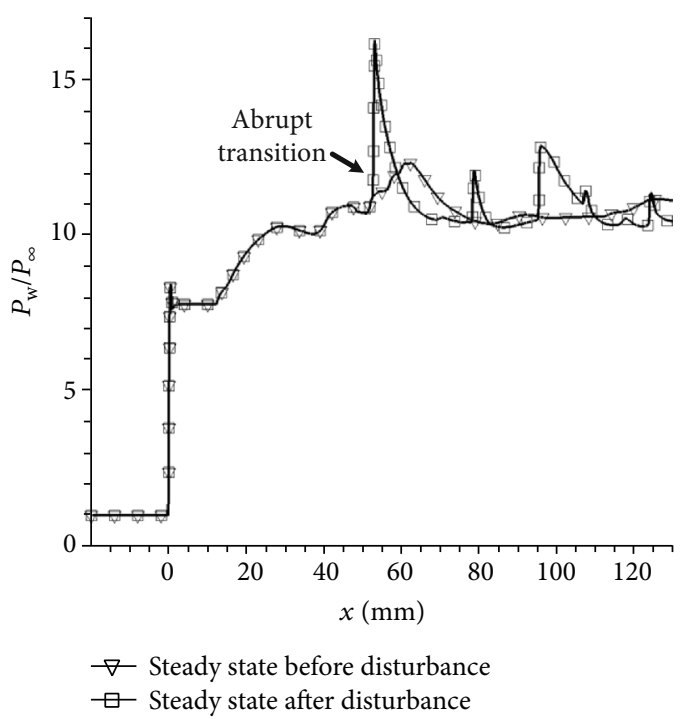

FIgURE 8: Pressure distributions on the bottom boundary of the steady flow fields before and after UTHD at $\theta=31^{\circ}$.

continuously oscillatory. After UTHD is introduced into the flow field, the pressure disturbance propagates downstream, and the flow field is disturbed significantly. A new triple point forms near the wedge tip (see Figure 2(c)) after the disturbance passes, and the ODW near this newly formed triple point is slightly bow-shaped. However, the triple point continuously moves downstream and the flow field finally recovers to very similar to its original state (compare Figure 2(a) and Figure 2(h)), except that the steady ODW after the disturbance has four steady triple points on its wave surface (denoted by TP1, TP2, TP3, and TP4, respectively, in Figure 2(h)). Thus, the primary triple point of the ODW is stable to the UTHD, but the wave surface is unstable to the UTHD.

To assess whether the aforementioned results are gridindependent, Figure 3 shows the steady ODWs before and after disturbed by UTHD for $64 \mathrm{pts} / \Delta I$. In both the undisturbed and disturbed cases, the results of $64 \mathrm{pts} / \Delta I$ are almost the same as that of $32 \mathrm{pts} / \Delta I$ (compare Figure 3(a) with Figure 2(a) and Figure 3(b) with Figure 2(h)), except that the slip lines of the $64 \mathrm{pts} / \Delta I$ are slightly clearer. Thus, 32 $\mathrm{pts} / \Delta I$ is sufficient to reach grid convergence in this study.

Considering that the primary triple point being stable to UTHD may be due to insufficient disturbance time, a longer-term DJFD disturbance is introduced into the original steady flow field at $\theta=32^{\circ}$. Figure 4 shows the evolution of the flow field after DJFD that lasts for $100 \Delta t$. Clearly, the evolution of the flow field takes longer compared to that of UTHD because the current disturbance is no longer transient. A significant bow shock together with a triple point forms immediately when the jet flow is introduced into the flow field (see Figure 4(a)). However, the triple point again moves continuously downstream. After sufficient time, the flow field becomes the same as that of the UTHD case (compare Figure 4(f) with Figure 2(h)). That is to say, for DJFD of $100 \Delta t$, the primary triple point is still stable. This indicates that the jet flow time may be still insufficient to trigger instability of the primary triple point.

Figure 5 shows the evolution of the flow field after DJFD of $200 \Delta t$ at $\theta=32^{\circ}$. A much stronger bow shock forms after the jet flow is introduced (compare Figure 5(a) with Figure 4(a)). After $36500 \Delta t$, which is sufficiently long, the primary triple point remains located somewhere upstream (near the wedge tip) and cannot recover to its undisturbed state (compare Figure 5(d) with Figure 2(a)). Furthermore, the ODW itself presents more unsteady features, including the unsteady slip line with large-scale vortexes and another unsteady triple point on the wave surface. Thus, for this longer-term jet flow, the primary triple becomes unstable. It seems that the formation of a strong bow shock or ODW near the wedge tip is the precondition for triggering instability of the primary triple point. Therefore, the effect of the location and intensity of the disturbance can be further deduced. That is, the primary triple point is stable if the location of the disturbance is significantly far from the primary triple point or if the intensity of the disturbance is insufficient to form a strong bow shock or ODW near the wedge tip. Figure 6 compares the pressure distributions on the bottom boundary of the steady flow fields after the $100 \Delta t$ - and 200 $\Delta t$-DJFD. Clearly, after the $200 \Delta t$-DJFD, there exists a remarkable pressure jump on the wedge surface, which indicates the reflection of the transverse shock. In the far downstream, the pressure on the wedge surface recovers at a similar level with the undisturbed case.

Now, we start to consider the case of $\theta=31^{\circ}$. Henceforth, the detailed evolution processes of the flow fields will not be provided since they are similar to the previous cases and we mainly focus on the steady flow fields before and after disturbances. Figure 7 shows the steady flow fields before and after UTHD of the $\theta=31^{\circ}$ case. Before UTHD, the ODW presents 


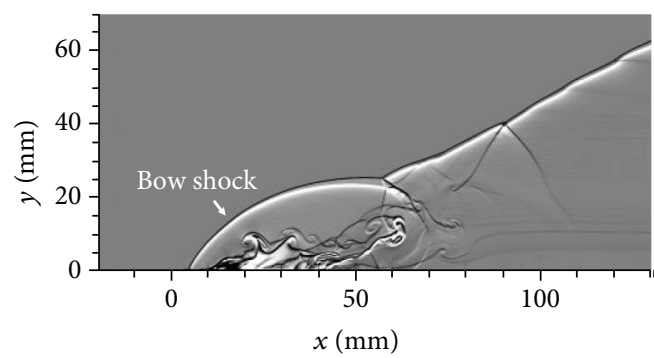

(a)

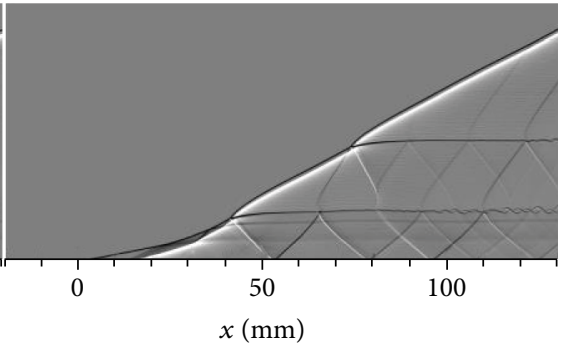

(b)

Figure 9: Evolution of the flow field after DJFD of $500 \Delta t$ at $\theta=31^{\circ}$. (a) $t=500 \Delta t$ (the jet flow has just been turned off) and (b) steady state after the disturbance.

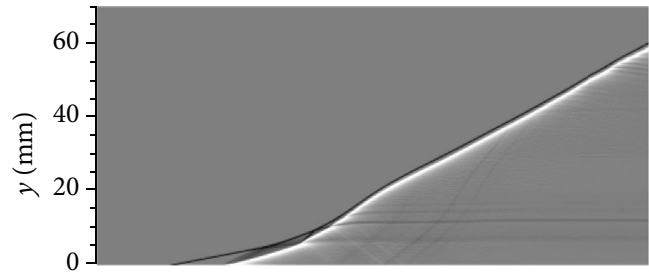

(a)

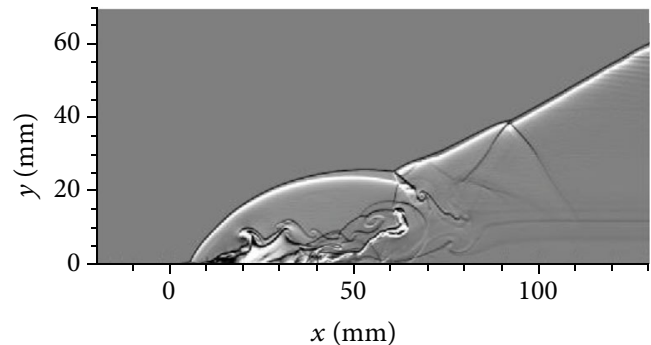

(b)

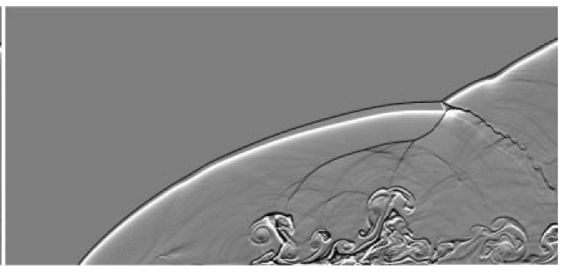

(c)

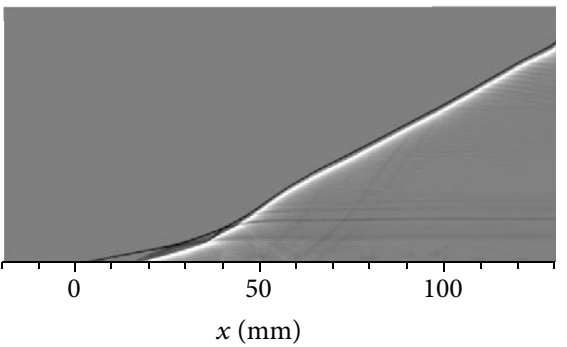

(d)

Figure 10: Evolution of the flow field after DJFD of $500 \Delta t$ at $\theta=30^{\circ}$. (a) $t=0$ (original steady state), (b) $t=500 \Delta t$ (the jet flow has just been turned off), (c) $t=700 \Delta t$, and (d) steady state after the disturbance.

a smooth transition pattern with no primary triple point or transverse shock. However, after UTHD, the transition pattern becomes abrupt, with the existence of the primary triple point and the transverse shock. Figure 8 compares the pressure distributions on the bottom boundary before and after UTHD. The transverse shock of the abrupt transition gives rise to a pressure jump on the wedge surface whereas the smooth transition has no sudden pressure jump.

Figure 9 shows the evolution of the flow field after DJFD that lasts for $500 \Delta t$ at $\theta=31^{\circ}$. Although the ODW is highly locally detached and bow-shaped near the wedge tip after the jet flow is turned off, as shown in Figure 9(a), it finally becomes the same as that after UTHD (compare Figure 9(b) with Figure $7(\mathrm{~b})$ ). Thus, there is no upstream moving triple point for the $\theta=31^{\circ}$ case.

Finally, the case of $\theta=30^{\circ}$ is tested. Figure 10 shows the evolution of the flow field after DJFD of $500 \Delta t$. It is seen that before and after the disturbance, the ODW keeps totally unchanged. Since the $500 \Delta t$-DJFD is a much more intense disturbance than UTHD, it is reasonable to expect that the ODW will still be unchanged by UTHD. This means that the ODW is absolutely stable to disturbances at $\theta=30^{\circ}$.
The stability of the steady ODW flow field after the disturbance is also worth discussing. At $\theta=32^{\circ}$, the steady flow field after UTHD or DJFD of $100 \Delta t$ (both can trigger wave surface instability) is stable to disturbance of the same type but remains unstable to DJFD of $200 \Delta t$ that can further trigger the primary triple point instability. However, the steady flow field after DJFD of $200 \Delta t$ is stable to any other kind of disturbances, suggesting that the ODW with an upstream primary triple point is absolutely stable, just like the case of $\theta=30^{\circ}$.

Although the ODW is unstable to flow disturbances in a certain wedge angle range, it can maintain standing stabilization on the wedge, which is beneficial to propulsive ODW applications.

\section{Conclusions}

The disturbance resistance of ODWs was numerically studied in this work using the two-dimensional reactive Euler equations with a two-step induction-reaction kinetic model. In contrast to previous studies by other researchers $[4,12$, 24-26], the present work demonstrates that an ODW is not 
stable or resistant to flow disturbances under certain conditions and is sensitive to the disturbance form. The ODW tends to be stable at a smaller wedge angle and unstable at a larger wedge angle. The unstable characteristics manifest themselves in the ODW structure and transition pattern. In the unstable wedge angle range, only the ODW surface and transition pattern change after UTHD and short-term DJFD, whereas the upstream moving of the primary triple point occurs after long-term DJFD because a local strong detached bow shock wave forms near the wedge tip via this kind of disturbance. Although the ODW presents unstable responses to disturbances, it maintains the standing stability after disturbances, which is desired for an oblique detonation engine.

\section{Data Availability}

The (numerical schlieren) and (pressure distribution) data used to support the findings of this study are included within the article.

\section{Conflicts of Interest}

The authors declare that there is no conflict of interest regarding the publication of this paper.

\section{Acknowledgments}

This work was supported by the National Natural Science Foundation of China (NSFC) (nos. 11702316 and 91641126).

\section{References}

[1] D. T. Pratt, J. W. Humphrey, and D. E. Glenn, "Morphology of standing oblique detonation waves," Journal of Propulsion and Power, vol. 7, no. 5, pp. 837-845, 1991.

[2] K. Ghorbanian and J. D. Sterling, "Influence of formation processes on oblique detonation wave stabilization," Journal of Propulsion and Power, vol. 12, no. 3, pp. 509-517, 1996.

[3] S. A. Ashford and G. Emanuel, "Wave angle for oblique detonation waves," Shock Waves, vol. 3, no. 4, pp. 327-329, 1994.

[4] C. Li, K. Kailasanath, and E. S. Oran, "Detonation structures behind oblique shocks," Physics of Fluids, vol. 6, no. 4, pp. 1600-1611, 1994.

[5] L. Í. S. F. Figueria da Silva and B. Deshaies, "Stabilization of an oblique detonation wave by a wedge: a parametric numerical study," Combustion and Flame, vol. 121, no. 1-2, pp. 152166, 2000.

[6] J. Y. Choi, D. W. Kim, I. S. Jeung, F. Ma, and V. Yang, "Celllike structure of unstable oblique detonation wave from highresolution numerical simulation," Proceedings of the Combustion Institute, vol. 31, no. 2, pp. 2473-2480, 2007.

[7] M. Gui and B. Fan, "Wavelet structure of wedge-induced oblique detonation waves," Combustion Science and Technology, vol. 184, no. 10-11, pp. 1456-1470, 2012.

[8] H. H. Teng and Z. L. Jiang, "On the transition pattern of the oblique detonation structure," Journal of Fluid Mechanics, vol. 713, pp. 659-669, 2012.

[9] J. Verreault, A. J. Higgins, and R. A. Stowe, "Formation and structure of steady oblique and conical detonation waves," AIAA Journal, vol. 50, no. 8, pp. 1766-1772, 2012.
[10] J. Verreault, A. J. Higgins, and R. A. Stowe, "Formation of transverse waves in oblique detonations," Proceedings of the Combustion Institute, vol. 34, no. 2, pp. 1913-1920, 2013.

[11] H. H. Teng, Z. L. Jiang, and H. D. Ng, "Numerical study on unstable surfaces of oblique detonations," Journal of Fluid Mechanics, vol. 744, pp. 111-128, 2014.

[12] Y. Liu, D. Wu, S. Yao, and J. Wang, “Analytical and numerical investigations of wedge-induced oblique detonation waves at low inflow Mach number," Combustion Science and Technology, vol. 187, no. 6, pp. 843-856, 2015.

[13] H. Teng, H. D. Ng, K. Li, C. Luo, and Z. Jiang, "Evolution of cellular structures on oblique detonation surfaces," Combustion and Flame, vol. 162, no. 2, pp. 470-477, 2015.

[14] P. Yang, H. Teng, Z. Jiang, and H. D. Ng, "Effects of inflow Mach number on oblique detonation initiation with a twostep induction-reaction kinetic model," Combustion and Flame, vol. 193, pp. 246-256, 2018.

[15] F. K. Lu, H. Fan, and D. R. Wilson, "Detonation waves induced by a confined wedge," Aerospace Science and Technology, vol. 10, no. 8, pp. 679-685, 2006.

[16] S. Bhattrai and H. Tang, "Formation of near-ChapmanJouguet oblique detonation wave over a dual-angle ramp," Aerospace Science and Technology, vol. 63, pp. 1-8, 2017.

[17] P. G. Harris, R. Farinaccio, R. A. Stowe, R. Link, D. Alexander, and L. Donahue, "Structure of conical oblique detonation waves," in 44th AIAA/ASME/SAE/ASEE Joint Propulsion Conference \& Exhibit, p. 4687, Hartford, CT, USA, 2008.

[18] J. Verreault and A. J. Higgins, "Initiation of detonation by conical projectiles," Proceedings of the Combustion Institute, vol. 33, no. 2, pp. 2311-2318, 2011.

[19] J. Kasahara, T. Arai, S. Chiba, K. Takazawa, Y. Tanahashi, and A. Matsuo, "Criticality for stabilized oblique detonation waves around spherical bodies in acetylene/oxygen/krypton mixtures," Proceedings of the Combustion Institute, vol. 29, no. 2, pp. 2817-2824, 2002.

[20] S. Maeda, R. Inada, J. Kasahara, and A. Matsuo, "Visualization of the non-steady state oblique detonation wave phenomena around hypersonic spherical projectile," Proceedings of the Combustion Institute, vol. 33, no. 2, pp. 2343-2349, 2011.

[21] S. Maeda, J. Kasahara, and A. Matsuo, "Oblique detonation wave stability around a spherical projectile by a high time resolution optical observation," Combustion and Flame, vol. 159, no. 2, pp. 887-896, 2012.

[22] S. Maeda, S. Sumiya, J. Kasahara, and A. Matsuo, "Initiation and sustaining mechanisms of stabilized oblique detonation waves around projectiles," Proceedings of the Combustion Institute, vol. 34, no. 2, pp. 1973-1980, 2013.

[23] S. Maeda, S. Sumiya, J. Kasahara, and A. Matsuo, "Scale effect of spherical projectiles for stabilization of oblique detonation waves," Shock Waves, vol. 25, no. 2, pp. 141-150, 2015.

[24] G. Fusina, J. P. Sislian, and B. Parent, "Formation and stability of near Chapman-Jouguet standing oblique detonation waves," AIAA Journal, vol. 43, no. 7, pp. 1591-1604, 2005.

[25] H. H. Teng, W. Zhao, and Z. L. Jiang, "A novel oblique detonation structure and its stability," Chinese Physics Letters, vol. 24, pp. 1985-1988, 2007.

[26] S. Miao, J. Zhou, Z. Lin, X. Cai, and S. Liu, "Numerical study on thermodynamic efficiency and stability of oblique detonation waves," AIAA Journal, vol. 56, no. 8, pp. 3112-3122, 2018.

[27] Y. Fang, Z. Hu, H. Teng, Z. Jiang, and H. D. Ng, "Numerical study of inflow equivalence ratio inhomogeneity on oblique 
detonation formation in hydrogen-air mixtures," Aerospace Science and Technology, vol. 71, pp. 256-263, 2017.

[28] P. Yang, H. D. Ng, and H. Teng, "Numerical study of wedgeinduced oblique detonations in unsteady flow," Journal of Fluid Mechanics, vol. 876, pp. 264-287, 2019.

[29] P. Yang, H. D. Ng, H. Teng, and Z. Jiang, "Initiation structure of oblique detonation waves behind conical shocks," Physics of Fluids, vol. 29, no. 8, article 086104, 2017.

[30] K. Wang, Z. Zhang, P. Yang, and H. Teng, "Numerical study on reflection of an oblique detonation wave on an outward turning wall," Physics of Fluids, vol. 32, article 046101, 2020.

[31] Y. Liu, L. Wang, B. Xiao, Z. Yan, and C. Wang, "Hysteresis phenomenon of the oblique detonation wave," Combustion and Flame, vol. 192, pp. 170-179, 2018.

[32] H. D. Ng, M. I. Radulescu, A. J. Higgins, N. Nikiforakis, and J. H. S. Lee, "Numerical investigation of the instability for one-dimensional Chapman-Jouguet detonations with chainbranching kinetics," Combustion Theory and Modelling, vol. 9, no. 3, pp. 385-401, 2005.

[33] P. Yang, H. Teng, H. D. Ng, and Z. Jiang, "A numerical study on the instability of oblique detonation waves with a two-step induction-reaction kinetic model," Proceedings of the Combustion Institute, vol. 37, no. 3, pp. 3537-3544, 2019.

[34] R. Deiterding, "Parallel adaptive simulation of multidimensional detonation structures, [Ph.D. thesis]," Brandenburgischen Technischen Universität Cottbus, 2003.

[35] J. Li, J. Ning, and J. H. S. Lee, "Mach reflection of a ZND detonation wave," Shock Waves, vol. 25, no. 3, pp. 293-304, 2015.

[36] J. Li and J. H. S. Lee, "Numerical simulation of Mach reflection of cellular detonations," Shock Waves, vol. 26, no. 5, pp. 673$682,2016$. 